\title{
SOURCE-NOISE IN RADIO SYNTHESIS IMAGES
}

\author{
K.R. ANANTHARAMAIAH, A.A. DESHPANDE AND \\ V. RADHAKRISHNAN \\ Raman Research Institute, Bangalore 560 080, India
}

\author{
R.D. EKERS \\ Australia Telescope, P.O. Box 76, Epping, NSW 2121, Australia \\ T.J. CORNWELL AND W.M. GOSS \\ NRAO, Very Large Array, P.O. Box 0, Socorro, NM 87801, USA
}

\begin{abstract}
We discuss the distribution of source- and receiver-noise in radio synthesis images and show that the source-noise is maximum at the position of the source but also appears in the off-source region because of the sidelobes. Analytical expressions are derived for the rms noise at any location of both "total-power" and "correlation" images. We show that under certain conditions, deconvolution can remove the source noise from the off-source region in snap-shot images. Some of the results are verified experimentally.
\end{abstract}

\section{INTRODUCTION}

There are two main contributions to the noise fluctuations that are present at the output of a radio telescope. The first, which we refer to as the receivernoise comes mainly from the electronics in the system. The second, which we refer to as the source-noise is due to the signal from the source itself which is similar in nature to the receiver-noise, but whose amplitude depends on the source strength. In a synthesis array, the receiver-noise of any two antennas are completely uncorrelated but the source-noise will be partially or fully correlated between the antennas depending on source structure and the baseline. Here we discuss the distribution of receiver- and source- noise in radio synthesis images. In general the receiver-noise is the dominant term and is expected to be uniform across the image since it is uncorrelated between the antennas. However, when the collecting area of the array is large or the source is strong, the contribution of the source-noise can be significant or even dominant. Since the correlation of source-noise is dependent both on baseline and source structure, the distribution of this noise in the image will have some relation to the array configuration and the source structure. The behaviour of source-noise in synthesis images has been discussed earlier by Anantharamaiah et al. (1989, hereafter Paper I) and Kulkarni (1989). An extension to the work of Kulkarni (1989) was presented at this meeting by Vivekanand and Kulkarni (this volume). 


\section{MAGNITUDE AND DISTRIBUTION OF NOISE}

Consider a radio image of a region around a strong source. If this image were made by taking scans across the region using a single-dish with good angular resolution and no sidelobes, then it is obvious that the noise in the off-source region is only due to the receiver noise and is given by $K_{1} S_{R} / \sqrt{B \tau}$, where $K_{1}$ is a constant dependant on the type of receiver, $B$ and $\tau$ have the usual meaning and $S_{R}$ is the noise equivalent flux density of the receiver obtained using $\frac{1}{2} S_{R} A_{e}=k T_{R}$, where $A_{e}$ is the effective area of the antenna and $T_{R}$ is the receiver noise temperature. On the source, the noise will be $K_{1}\left(S_{R}+S_{b}\right) / \sqrt{B r}$, where $S_{b}$ is the source brightness (flux/beam). Therefore the source-noise contributes only at the position of the source and nothing to the off-source region.

In a synthesized image of the same region made with a dilute array of $N$ elements, however, the source-noise does contribute to the off-source region. As discussed in Paper $I$, this is a consequence of the sidelobes created by the "unfilled" nature of the synthesis array. The net off-source noise can be as much as $K_{2}\left(S_{R}+S\right) / \sqrt{N_{b} B \tau}$, where $S$ is the total flux density of the source and $N_{b}=N(N-1) / 2$ is the number of baselines. $\left(S_{R}+S\right)$ reperesents the total system noise at each of the antennas. Since the presence of the source-noise in the off-source region is due to the instantaneous sidelobes of the array (Paper I), it might appear that a proper deconvolution may be able to remove this noise from the off-source region and leave it only at the position of the source, as in the case of a single-dish image. This is in fact true, but only in the case of a snap-shot image of a unresolved source with no receiver noise. In this case the off-source noise has exactly the same structure as the dirty beam and is fully correlated between the different pixels of the image. Deconvolution will reduce the off-source noise to zero and the on source noise will have the usual value of $S / \sqrt{B \tau}$. Note that, on the source, the fact that there are N elements in the array has no effect on the noise since the source-noise (which is the only source of noise in this case) is fully correlated between the antennas. However, when receiver noise is present, or when the source is resolved, deconvolution cannot reduce the off-source noise to the value given only by the receiver-noise, i.e. $S_{R} / \sqrt{N_{b} B \tau}$, but will have an additional cross term either between the source- and the receivernoise or between different parts of the source. The on-source noise will have a third term of the order $S_{b} / \sqrt{B \tau}$.

When the image is made using full synthesis, which is a combination of a series of snap-shots, then, as discussed in Paper I, the distribution of source-noise in the off-source region has no relation to the synthesized beam. This is because the synthesized beam is a linear combination of the snap-shot beams, whereas, since the source-noise (as also the receiver-noise) is independent in different snap-shots, the final noise is the square-root of the sum of the squares of the noise in individual snap-shots. Therefore any deconvolution cannot remove the source-noise from the off-source region. It is easy to see that the magnitude of the source-noise in the off-source region should be proportional to the rms sidelobe level of the individual snap-shots. The rms sidelobe levels is $\sim S / \sqrt{N_{b}}$ and follows either directly from Parseval's theorm or if we look at the off-source level as resulting from addition of $N_{b}$ random vectors (random in phase) each of amplitude $S$. The net source-noise in the off-source region is therefore $\sim \frac{\left(S / \sqrt{N_{b}}\right)}{\sqrt{B T}}$. The receiver noise which is independent between the antennas has the usual 
value $S_{R} / \sqrt{N_{b} B \tau}$. It is interesting that the contribution of both the sourceand the receiver- noise to the off-source region has identical dependence on $N_{b}$, although the reasons are somewhat different. The actual dependence of sourcenoise term on $N_{b}$ can be different depending on the array configuration. As mentioned above, in addition to these two terms, there will be a cross term of the form $\sim \sqrt{S . S_{R} /(N B \tau)}$ which contributes to the off-source noise. The total off-source noise will be a combination of the squares of these three terms. If the cross-term has a coefficient $\sqrt{2}$ (which as we show later is true only for "totalpower" interferometers), then the sum of the squares of the three terms will be a perfect square and the rms off-source noise will be $\left(S_{R}+S\right) / \sqrt{N_{b} B \tau}$, which is the usual expression used for noise in synthesis images (e.g. Crane and Napier 1989). Note that this only applies to the off-source regions of the image. On the source, the source-noise term will not have the factor $\sqrt{N_{b}}$, since the noise is fully (or partially) correlated between the antennas. The net on source noise should therefore be of the order $\frac{1}{\sqrt{B T}}\left(S^{2}+S . S_{R} / N+S_{R}^{2} / N_{b}\right)^{1 / 2}$.

In order to get a general and more precise quantitative expression for the noise in different parts of the image we consider an array of $\mathrm{N}$ elements which can be "phased" to point anywhere within the primary beam of the individual antennas. We only present an outline of the analysis here and the details will be published elsewhere. The total output voltage of the phased-array for any direction $\left(l_{0}, m_{0}\right)$ is given by

$$
\Psi\left(l_{0}, m_{0}\right)=\frac{1}{N} \sum_{i=1}^{N}\left(\Psi_{i}+\eta_{i}\right)
$$

where $\Psi_{i}$ is the complex voltage due to the source at antenna $i$ and $\eta_{i}$ is the voltage due to the receiver-noise. If $\left(x_{i}, y_{i}\right)$ is the location of the antenna $i$ then

$$
\Psi_{i}=\sum_{l, m} \Psi_{0}(l, m) \exp \left\{-j 2 \pi\left[x_{i}\left(l-l_{0}\right)+y_{i}\left(m-m_{0}\right)\right]\right\}
$$

where $\Psi_{0}(l, m)$ is the complex voltage received at $(x, y)=(0,0)$. If we consider a "total-power" image, then the average value of the image at any location is given by

$$
\left\langle S\left(l_{0}, m_{0}\right)\right\rangle=\left\langle\Psi\left(l_{0}, m_{0}\right) \cdot \Psi^{*}\left(l_{0}, m_{0}\right)\right\rangle
$$

In order to get the error in this quantity (which is the noise in the image) we expand $S\left(l_{0}, m_{0}\right)$ as a Taylor series near the expectation value of $\Psi_{0}(l, m)$ and get

$$
\left\langle\Delta S^{2}\right\rangle=\sum_{i}\left\langle\left(\frac{\delta S}{\delta P_{i}}\right)^{2}\right\rangle\left\langle d P_{i}^{2}\right\rangle
$$

where the parameters $P_{i}$ are the real and imaginary parts of the receiver and source noise voltages $\eta_{i}$ and $\Psi_{i}$ and which are normal random variables with zero mean. Full computation of equation (4) leads to the following simple and elegant expression for the rms noise at any loction $\left(l_{0}, m_{0}\right)$ in a snap-shot "total-power" image.

$$
\Delta S_{r m s}=\frac{1}{\sqrt{B \tau}}\left[S_{o b s}\left(l_{0}, m_{0}\right)+\frac{S_{R}}{N}\right]
$$


$S_{o b s}\left(l_{0}, m_{0}\right)$ is the observed value in the dirty image at the position $\left(l_{0}, m_{0}\right)$, which for the off-source region is merely the strength of the sidelobe at that location. This is consistent with the arguments presented in Paper I where it was concluded that the off-source noise must be proportional to the sidelobe level. Since this expression does not have cross terms of the form $\left(S . S_{R}\right)$, it immediately suggests that deconvolution of a snap-shot "total-power" image can completely remove the source-noise from the off-source region. The off-source region will then have only the receiver noise as in the case of a single-dish image. The extension of eqution (5) for a full-synthesis image is straight forward.

In practice radio synthesis images do not include the total power but only the correlation between the antennas. In this case the average value of the image at any location is given by

$$
\left\langle S\left(l_{0}, m_{0}\right)\right\rangle=\frac{1}{N(N-1)}\left[\Psi \cdot \Psi^{*}-\sum_{i=1}^{N}\left(\Psi_{i}+\eta_{i}\right) \cdot\left(\Psi_{i}^{*}+\eta_{i}^{*}\right)\right]
$$

where the second term on the right represent the total powers of the $\mathrm{N}$ antennas. Following similar procedure we have obtained an expression for the rms noise at any position in the image. This expression is more complicated than equation (5), contains cross terms of the form $\left(S_{o b s}\left(l_{0}, m_{0}\right) . S_{R}\right)$ and is not presented here for lack of space. Although complicated, the different terms of this expression lend themselves to physical interpretation and these will be discussed elsewhere. For the special case of an unresolved source of flux density $\mathrm{S}$, it reduced to $\Delta S_{\text {on }}=\frac{1}{\sqrt{B \tau}}\left(S^{2}+2 S S_{R} / N+S_{R}^{2} / N(N-1)\right)^{\frac{1}{2}}$ for the on-source noise and $\Delta S_{o f f}=\frac{1}{\sqrt{N_{b} B \tau}}\left[\left(S+S_{R} / \sqrt{2}\right)^{2}+S S_{R}\right]^{\frac{2}{2}}$ for the off-source noise. The corresponding total-power expressions are $\Delta S_{o n}=\frac{1}{\sqrt{B \tau}}\left(S+S_{R} / N\right)$ and $\Delta S_{\text {off }}=\frac{1}{N \sqrt{B \tau}}\left(S+S_{R}\right)$.

It is well known that in the limit of large $N$, the difference between a total power and a correlation image reduces and therefore it is our hope that the elegant expression given in equation (5) can be used to describe the distribution of noise in any radio synthesis image.

Some of the results presented above were verified experimentally using data taken at $90 \mathrm{~cm}$ in the D-configuration of the VLA towards the strong source Cas A. We find that the noise distribution in a snap-shot image follows the sidelobe pattern as suggested by equation (5). Deconvolution using 'clean' decreased the magnitude of this noise as predicted. The noise distribution in the full synthesis image has no correspondence with the synthesized dirty beam and its magnitude is unaltered by deconvolution. More details will be presented elsewhere.

\section{REFERENCES}

Anantharamaiah, K.R., Ekers, R.D., Radhakrishnan, V., Cornwell, T.J. and Goss, W.M. 1989, in Synthesis Imaging in Radio Astronomy, ed. R.A. Perley, F.R. Schwab and A.H. Bridle, Pub. A.S.P., 6, p431

Crane, P.C. and Napier, P.J. 1989 in Synthesis Imaging in Radio Astronomy, ed. R.A. Perley, F.R. Schwab and A.H. Bridle, Pub. A.S.P., 6, p139

Kulkarni, S.R. 1989, Astron. J., 98, p1112 